\title{
Verbal predictions of unexpected stimuli and choice reaction time
}

\author{
MICHAEL J. HACKER \\ Rockefeller University, New York, New York 10021 \\ and \\ JAMES V. HINRICHS \\ University of Iowa, Iowa City, Iowa 52242
}

\begin{abstract}
In two choice reaction time (RT) experiments, subjects verbally predicted the stimulus alternative that was either most or least likely to be presented on each trial. An expectancy interpretation of prediction effects requires that stimuli matching least likely predictions yield the longest RTs. In Experiment 1, with four stimulus alternatives, RTs to stimuli matching the verbalized stimulus, both most likely and least likely predictions, were shorter than RTs to stimuli that matched neither the most nor the least likely predictions. However, matching most likely predictions produced faster responses with two alternatives. In Experiment 2, multiple predictions of both most and least likely stimuli were compared with joint predictions of first and second most likely alternatives. RTs to stimuli matching least likely predictions were longer than RTs to most likely matches, implicating prediction type as a partial determinant of RT. Matching least likely stimuli were responded to more quickly than unpredicted alternatives, demonstrating the importance of generating a prediction per se over the implied level of expectancy. Results from both experiments were contrasted with nonparametric predictions from an ordered memory scanning model.
\end{abstract}

Choice reaction times (RTs) to correctly predicted stimuli are faster than choice RTs to incorrectly predicted stimuli, perhaps approaching simple RT as a lower bound (cf. Bernstein \& Reese, 1967). Although verbal predictions have been employed as a means of controlling for subjective expectancies in the assessment of repetition and frequency effect (e.g., Hinrichs, 1970; Keele, 1969; Williams, 1966), it has never been demonstrated that the different RTs associated with correct and incorrect prediction outcomes are in fact a consequence of expectations revealed by verbal predictions as opposed to attentional states either correlated with or induced by the prediction process.

A strong expectancy interpretation, such as the continuous expectancy theory of Geller and Pitz (1970), requires not only that responding to subjectively likely stimuli be facilitated, but also that responding to stimuli at the other end of the expectancy continuum be inhibited (cf. Geller, 1975). There appears to be no a priori reason why subjects could not report items from either end of the continuum. An attention interpretation, while not denying that subjective expectancies may mediate the production of verbal predictions,

The authors thank Dale Yurko for his assistance in running subjects in Experiment 1. Publication of this research was partially supported by NIMH Grant 15125 . Requests for reprints should be sent to Michael J. Hacker, William James Hall, Harvard University, Cambridge, Massachusetts 02138. assumes that the functional properties of an overt prediction are similar to those of an experimentally controlled cue or prime. The processing required in the generation and verbalization of a potential stimulus, "expected" or otherwise, may evoke an attentional shift to that stimulus and hence reduce the identification latency. Whether or not such a shift occurs should depend upon the logical implications of the prediction instructions and other task characteristics such as processing load. Closely related to these two interpretations is the fundamental question: Are prediction effects a function of subjective stimulus expectancies, or are they merely an artifact of the prediction process? This question was investigated in the present study by instructing subjects to predict either the most likely stimulus or the least likely stimulus prior to each trial in a choice RT task.

If the shorter RTs following correct predictions are in fact a consequence of subjective expectancy, then the identification of stimuli matching "least likely" predictions should not be facilitated; in the context of the model presented below, there should be a predictable increment in choice RT. In contrast, the premise that overt predictions reveal and thus control for subjective expectancies, which are presumed to be influential even when they have no objective basis (e.g., Keele, 1969), may be in error. If in fact the production of a prediction per se automatically diverts attention to the predicted stimulus, then the identification of a stimulus 
overtly designated as unlikely should nevertheless be facilitated, provided that there are no conflicting stimulus cues.

The model we have most recently favored is an adaptation of a pushdown stack, serial memory scanning model first proposed by Theios and Falmagne (Note 1). This model, which closely resembles a better known model by Theios, Smith, Haviland, Traupmann, and Moy (1973), is characterized by the assumption of a self-terminating scanning stage, as opposed to the more common assumption of an exhaustive scan advanced by Sternberg (1966). Theios and Falmagne (Note 1) proposed a correspondence axiom by which an ordered set of subject-generated stimulus predictions is isomorphic with the scanning order of S.R representations in active memory. This relationship is presented schematically in Figure 1.

Given that identification is based on a serial, selfterminating scan of the hypothesized memory stack, the prediction effect follows from the implication that the stimulus predicted to be most likely is also the stimulus component of the first $S \cdot R$ element to be scanned. If the predicted stimulus matches the presented stimulus, the scan self-terminates after a single comparison, resulting in an appreciable savings in processing time. Since the complete structure of the memory stack is available for the generation of predictions, it also follows that the presentation of a stimulus matching the last element of a complete prediction set, or a single "least likely" prediction, would be followed by an exhaustive scan and, hence, a maximum RT. More importantly, the model makes precise nonparametric predictions as to the magnitude of prediction effects as a function of prediction order and set size.

Theios and Falmagne (Note 1) did not test their prediction axiom; however, Hacker and Hinrichs (1974) provided a partial test in a study of multiple-prediction effects. Specifically, subjects were instructed to report which two of four stimuli they felt to be first and second most likely to be presented. Consistent with the scanning model, mean RT was linearly related to expected memory stack position as inferred from stimulus predictions. In the first of the two experiments that follow, the relative accessibility of terminal stack entries as a function of memory set size was evaluated.

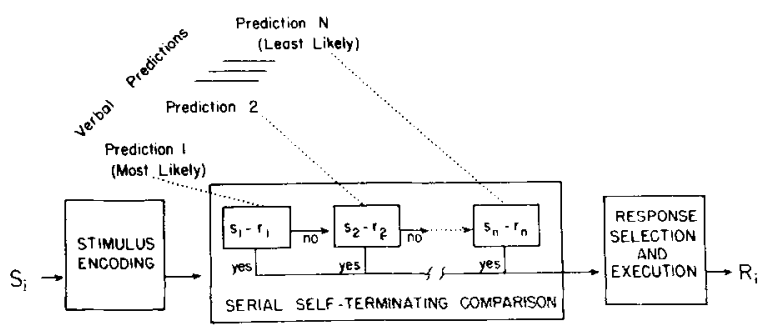

Figure 1. Hypothetical relationship between verbal predictions and ordered memory scanning.

\section{EXPERIMENT 1}

\section{Method}

Subjects. The subjects were 24 right-handed female students not more than 25 years old. Participation earned laboratory credit in an introductory psychology course.

Design and Procedure. Prediction type was counterbalanced so that half of the subjects initially predicted the most likely stimulus and then switched to predicting the least likely stimulus, while the remaining subjects proceeded in reverse order. Within each order, half of the subjects were assigned to a twostimulus group in which one stimulus was mapped to each of the two response alternatives available under each condition. The other half of the subjects were assigned to a four-stimulus group in which two stimuli were mapped to each response.

The stimulus letters $B, J, Q$, and $X$ were those previously selected by Hacker and Hinrichs (1974) on the basis of a number of criteria, particularly low visual and auditory confusability. The left-right response assignments for the six subjects receiving either of the two presentation orders for prediction type and two stimuli were B-J, Q-B, B-X, Q-J, J-X, and X-Q. For the four-stimulus conditions, the assignments were $B J-Q X, J Q-X B$, BX-JQ, QB-XJ, XJ-BQ, and XQ-BJ. Each prediction type was tested within a block of 200 trials. Equal numbers of each relevant stimulus were randomly permuted within five consecutive subblocks of 40 trials. Data from the first subblock under both prediction types were excluded from the subsequent analyses. Different random sequences were used for each of the $12 \mathrm{~S}-\mathrm{R}$ assignments listed above.

The two major trial blocks were separated by approximately 5 min during which the apparatus was reset and instructions appropriate to the shift in prediction type were read to the subject. The importance of formulating all predictions on a trial-by-trial basis was emphasized, consistent with the instructional set that most reliably yields matching in probability learning studies (Estes, 1964). Finally, the subject was instructed to respond as quickly as possible without making errors.

Either response terminated the stimulus presentation and initiated a 3 -sec intertrial interval, which was bisected $1.5 \mathrm{sec}$ later by the onset of a $200-\mathrm{msec}$ warning tone. The tone allowed subjects to pace their predictions. There was no explicit feedback regarding RT or accuracy.

Apparatus. Stimuli were presented on a Monsanto MDA III alphanumeric display assembly that was mounted at eye level, approximately $60 \mathrm{~cm}$ in front of the seated subject. Responses were made by pressing one of two labeled response buttons positioned $38 \mathrm{~cm}$ below and $13 \mathrm{~cm}$ to the left or right of the stimulus display unit. Stimulus presentations and the collection of response latencies to the nearest millisecond were controlled by a solid state logic system. Predictions were hand recorded by the experimenter.

\section{Results and Discussion}

After excluding response errors and RTs over $2 \mathrm{sec}$, each individual's RTs were sorted according to the identity of the presented and predicted stimuli. Mean RTs were computed for each condition and then averaged across specific stimuli to yield four experimental conditions. These conditions represented the cross of two factors, prediction type (most vs. least likely), and prediction outcome (match vs. mismatch). Note that a matching outcome for a least likely prediction implies that a stimulus predicted to be least likely was actually presented (hence matched the presentation) and not that the least likely prediction was verified by the nonoccurrence of the predicted stimulus. 
A four-way analysis of variance of the mean RTs (type by outcome by order by set size; $\alpha=.01$ for all analyses) indicated significant main effects for prediction type $[\mathrm{F}(1,20)=24.72, \mathrm{MSe}=1,417]$ and prediction outcome $[F(1,20)=122.01, \mathrm{MSe}=2,181]$. Neither the main effect for order nor its interactions achieved significance. The mean RTs collapsed across order are plotted in Figure 2. Although set size was not a significant factor by itself, it entered into significant interactions with both prediction type $[\mathrm{F}(1,20)=29.24$, $\mathrm{MSe}=63,757]$ and prediction outcome $[\mathrm{F}(1,20)=$ $9.06, \mathrm{MSe}=12,834]$. No other effects achieved significance.

Separate within-subjects analyses of the two- and four-stimulus conditions indicated substantial prediction type and Type by Outcome effects $[F(1,10)=22.85$, $\mathrm{MSe}=1,976$, and $\mathrm{F}(1,10)=12.84$, MSe $=2,130$, respectively] for the two-stimulus condition but no effects for the four-stimulus condition $[\mathrm{F}(1,10)=3.18$, MSe = 859 , and $F(1,10)=6.53$, $\mathrm{MSe}=601$, respectively $]$.

From Figure 2 and the preceding analyses, it is apparent that stimulus set size is an important determinant of the magnitude, if not the direction, of the prediction effect. Under standard "most likely" instructions, a 101-msec effect was observed for two stimulus alternatives. The 175 -msec effect for four stimuli was significantly larger, although less than the $100 \%$ increase predicted by the Theios and Falmagne (Note 1) model. Assuming a self-terminating search among four equally likely alternatives, and further assuming that the search begins with an incorrectly predicted (nonmatching) item, an average of two of the remaining three items must be scanned in order to respond correctly. With

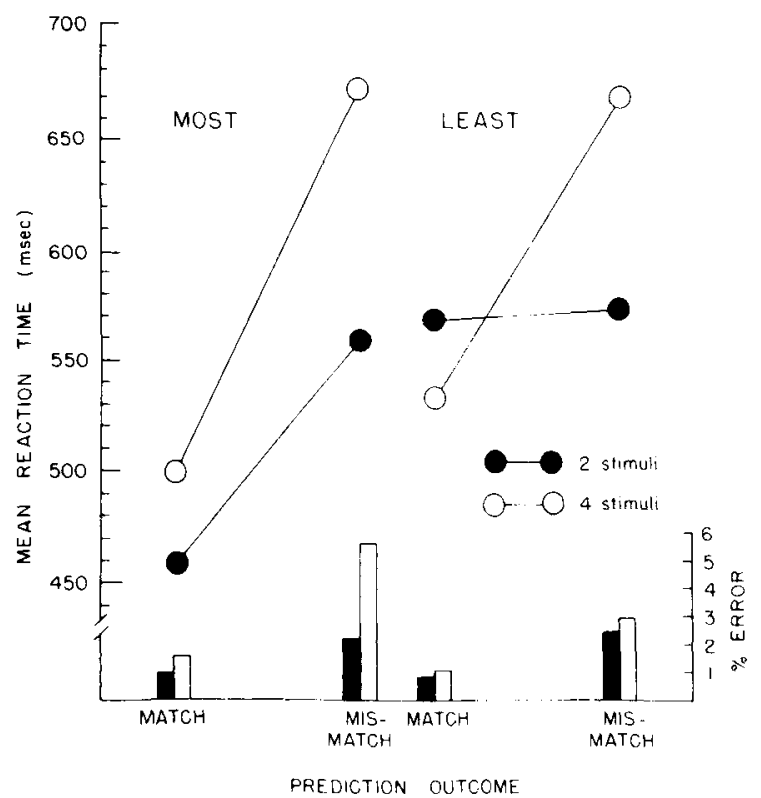

Figure 2. Comparison of decision times following verbal predictions of most likely and least likely stimuli as a function of set size and prediction accuracy. only two stimulus aiternatives, there is only one extra item to scan after a mispredicted item has been eliminated. Although the increment in the prediction outcome effect for four alternatives was $25 \mathrm{msec}$ less than the $200 \mathrm{msec}$ predicted by the preceding analysis, it should be noted that Shiffrin and Schneider (1974) categorically rejected all self-terminating scanning interpretations of the prediction effect on the assumption that expectancy effects do not increase with set size. Their assumption is clearly at odds with the present data.

For least likely predictions, the picture is somewhat altered. As anticipated, the largest outcome effect was obtained with four stimulus alternatives, but the direction of the effect was inconsistent with a simple expectancy interpretation. The RTs were shortest when the stimulus designated as least likely matched the presented stimulus; that is, the fastest responding occurred when subjects were ostensibly incorrect. With the larger stimulus set, the semantic sense of the prediction is apparently irrelevant, "most" being effectively equivalent to "least." This is interpreted as evidence of production rather than expectancy control.

With least likely predictions and only two stimulus alternatives, there was no significant prediction effect. As often happens, unexpected null results invite the most exotic explanations. One tenuous interpretation is that the lack of an effect reflects a partial tendency to reverse the attentional or reordering effects associated with prediction generation and overt verbalization. In the four-stimulus case, the specification of one stimulus as least likely has no differential implications for the three alternatives, so that the focus remains on the verbalized stimulus. With only two stimuli, say $B$ and $Q$, asserting that $\mathrm{B}$ is least expected logically implies that $\mathrm{Q}$ is most expected. This implication may suffice on some trials or for some subjects to redirect attention to the alternative ("expected") stimulus. In fact, a small but significant reversal was obtained for the two-stimulus condition in an earlier pilot study. ${ }^{1}$ It may be possible to increase the effect by stressing the logical implications of least likely predictions for alternative stimuli as part of the instructional set.

Although a probabilistic mixture of scanning orders might allow for the failure to obtain a prediction outcome effect in the least likely, two-stimulus condition, a mixture cannot account for the overall increase in RT associated with least likely predictions. This increase, which is not evident in the four-stimulus condition, necessarily reflects an unexplained increment in a processing stage other than a self-terminating scan. Averaged across matches and mismatches, the expected number of elements scanned (and hence the contribution of the scanning stage to the RTs) is identical for both prediction types, regardless of the order in which the unexpected stimuli are processed.

A difficulty related to the preceding problem of inferring which stimuli are expected from reports of 
unexpected stimuli is the more basic question of whether an expectancy construct has validity in the absence of objective stimulus information, and, if so, whether it is possible to specify the antecedents of such irrational expectations. Models of covert expectancy shifts (e.g., Theios et al., 1973), as well as the standard models of overt prediction embodied in the probability learning literature (see Estes, 1964, for a review), usually link the development of expectancy states or subjective probabilities to trial-by-trial variations in the stimulus sequence and only secondarily to the overall relative frequencies. There is considerable evidence from prediction protocols and RTs that subjects are more influenced by recent stimulus presentations than by relative frequency, that there is a strong tendency toward probability matching, and that RTs conditionalized on prediction outcome show little if any residual effect of stimulus frequency (Geller \& Pitz, 1970; Keele, 1969; Hacker \& Hinrichs, 1974; Hinrichs, 1970; Hinrichs \& Craft, 1971).

If neither the development of trial-by-trial expectations nor the magnitude of prediction outcome effects upon RT is a direct function of stimulus frequency, then the results obtained with equally likely stimuli in the present study are theoretically generalizable to conditions in which stimulus probabilities are varied. Furthermore, if the present design has generality, then the fact that subjects willingly, sometimes enthusiastically, persist in their attempts to predict unstructured stimulus strings provides an ideal context in which to evaluate the effects of generating most and least likely predictions unconfounded by external sources of stimulus information.

Further analysis of the present experiment was concerned with the possibility that the prediction outcome effects might result from a simple response bias or motor facilitation, as opposed to a selective stimulus set. The four-stimulus two-response condition provides a reduction paradigm in which it is possible for the stimulus prediction to correspond to the correct response but not to the correct stimulus (cf. Hinrichs \& Krainz, 1970). The mismatching prediction RTs from the fourstimulus condition were repartitioned into response matches and response mismatches. Averaging across individual subject means, the RTs for response matches and mismatches, given a most likely prediction, were 674 and $678 \mathrm{msec}$, respectively. For a least likely prediction, the corresponding values were 672 and $678 \mathrm{msec}$. Neither the main effects of prediction type and response prediction outcome nor their interaction was significant (all $\mathrm{Fs}<1.00$ ). Clearly, any response facilitation related to prediction outcome depended upon exact stimulus specification; no residual response bias was evident.

Finally, the mean percentages of response errors within each condition are included in Figure 2. Conditionalized error rates are seldom reported for verbal prediction effects, but overall error rates are commonly less than $4 \%$. If error rates are low and positively correlated with RT, it is often assumed that, at the very least, ordinal RT effects are not confounded by a potential speed-accuracy tradeoff (see Wickelgren, 1977, for a critique of this position). Although there were too few errors in the present study to support standard analyses, there does appear to be a direct relationship between RT and error rate. However, were it not for the stimulus reduction results noted above, a positive correlation between speed and accuracy would also be consistent with the possibility that stimulusindependent, prediction-oriented fast guesses (cf. Yellot, 1971) are a substantial component of the prediction outcome effect. Unfortunately, with one-to-one S-R mappings, there is no assurance that this is not the case, and the role of response accuracy is one that needs to be pursued in the prediction literature.

\section{EXPERIMENT 2}

Since Hacker and Hinrichs (1974) previously established the differential effects of first-vs. second-choice prediction outcomes in a four-stimulus design, it is of some interest to discover why most and least likely prediction outcomes were equivalent for the fourstimulus condition in the present experiment. One major procedural difference involved the production of both prediction types within a single trial in the initial Hacker and Hinrichs study. It may be that the specification of a first or most likely stimulus provides a context emphasizing the semantic implications of higher order predictions and disrupting the tendency to shift attention indiscriminantly to whatever stimulus is verbalized. Experiment 2 was designed to evaluate the most-least effects within a double-prediction context.

\section{Method}

Twenty-four additional subjects were recruited from the same pool sampled in Experiment 1. Half of the subjects were assigned to a group required to predict first and second most likely stimulus pairs, and the other half, to a group required to predict most-least pairs. All subjects received the same set of four stimulus letters (B, J, Q, X). With 12 subjects in each group and two stimuli assigned to each of two response alternatives, it was possible to counterbalance the $S-R$ assignments completely.

Following a 40-trial practice block, each subject received two 144-trial test blocks. There was a rest period between trial blocks, but the instructions were fixed within the entire experimental session. The intertrial interval was extended to $4 \mathrm{sec}$, with a $2-\mathrm{sec}$ foreperiod from the warning tone to stimulus onset in order to allow more time for the production of prediction pairs. Order within prediction pairs was fixed so that the first member of the pair always referred to the first-choice or most likely stimulus and the second member designated either the second-choice or least likely stimulus. All other aspects of the methodology including the apparatus were identical to Experiment 1.

\section{Results and Discussion}

Excluding errors and RTs over $2 \mathrm{sec}$, the individual RTs for each block were partitioned according to the three possible prediction outcomes (most or first predic- 
tion match, least or second prediction match, and no match). The means of the subject means, collapsed across blocks for the two types of prediction pairs, are plotted as a function of expected memory stack position in Figure 3.

The first-second prediction data clearly replicated Hacker and Hinrichs' (1974) previous observation of a linear relationship between inferred scanning order and RT. In the earlier study, the slope and intercept (leastsquares solution) were 64 and $492 \mathrm{msec}$, respectively. The comparable values for the present study were 52 and $500 \mathrm{msec}$. In contrast with the obvious linearity of first-second prediction data, the most-least means were nonmonotonic with expected memory stack position. However, unlike Experiment 1, in which most and least likely prediction outcomes could not be distinguished, stimuli matching the least likely component were responded to $88 \mathrm{msec}$ more slowly than stimuli matching the most likely component. Indeed, least likely matches appeared to be most comparable to second-choice matches.

In order to evaluate the effects of practice block and prediction outcome, as well as to compare prediction type, an analysis of variance was performed in which the three levels of outcome (within each of the two prediction types) were determined by the order in which the predictions were verbalized, rather than by expected scanning order. Thus, most and first prediction matches were associated with the first level, least and second prediction matches, with the second level, and nonmatches, with the third level. If the effect of a least likely prediction deviated significantly from that of a second choice, this would have been apparent in the

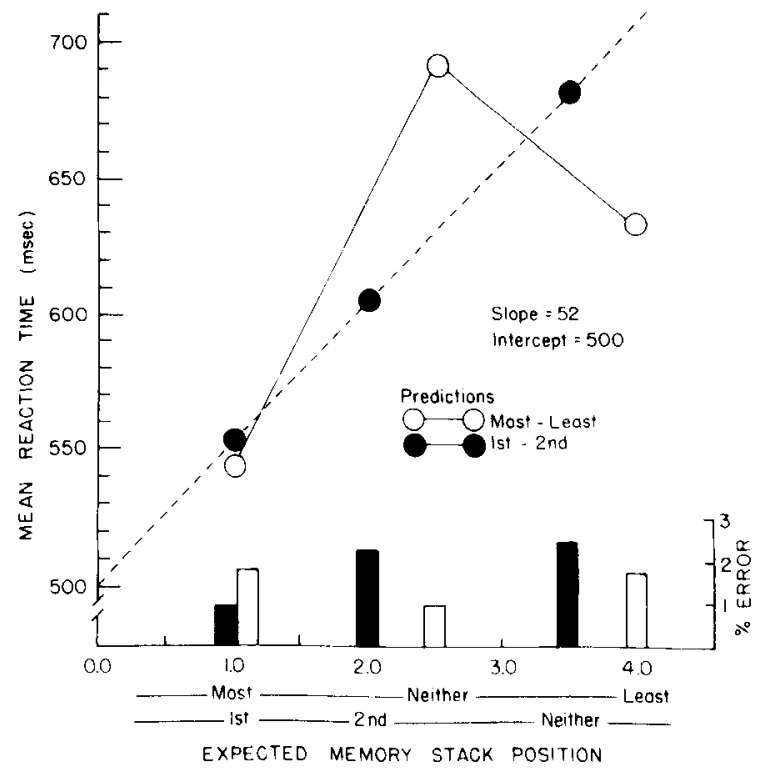

Figure 3. Choice reaction time as a function of prediction type and hypothetical memory position. Note that the expected position for unpredicted most-least comparisons is 2.5 and for unpredicted first-second comparisons is $\mathbf{3 . 5}$. interaction of prediction type and outcome. The only significant effects obtained were the main effects of prediction outcome and practice blocks $[\mathrm{F}(2,44)=$ 103.92, $\mathrm{MSe}=2,250$, and $\mathrm{F}(1,22)=22.52, \mathrm{MSe}=4,049$, respectively]. The Prediction Type by Prediction Outcome interaction was not significant $[F(2,44)=1.45]$, and all other effects produced $F$ ratios less than 1 . Hence, least likely predictions could not be distinguished from second most likely predictions in Experiment 2, just as they were indistinguishable from most likely predictions (with only one prediction of the four alternatives) in Experiment 1. The correspondence of the two prediction types in the present experiment can be further illustrated by replotting the most-least data in Figure 3 so that the mean RTs are a function of the same expected memory stack positions assigned to the first-second data. The independent estimates of the slope and intercept for the replotted data are 58 and $499 \mathrm{msec}$, respectively, which are very close to the values reported for the first-second condition.

\section{GENERAL DISCUSSION}

If the Theios and Falmagne (Note 1) prediction/ scanning order axiom fails to provide a complete account of the present data, is it possible that RT is determined directly by the production of predictions rather than both predictions and RTs being codetermined by internal states? If this is the case, there are still a number of important constraints that must apply to such a result. One significant implication that follows from the multiple-prediction studies is that the overt verbalization of the predicted stimulus (a potential auditory prime) is not per se the controlling factor. Recall that both second and least likely choices were always the more recent components of the prediction pairs. On the basis of any simple trace interpretation, the most recent event should have had the greatest effect, but this was not the case.

There is also recent evidence that the generation of a prediction is more important than its overt production. Acosta (1977) compared verbal prediction with a yoked control group required to read and then to verbalize predictions provided by the experimental group. For two stimulus alternatives, the maximum effect of such noninformative, nongenerative cuing was only $30 \mathrm{msec}$, compared with a 114-msec prediction effect for the same 500 -msec prediction-to-stimulus interval. Furthermore, there was no effect of a provided cue for a longer 4-sec interval, while the effect of subjectgenerated predictions was still substantial (about $80 \mathrm{msec}$ ).

Serial scanning may still provide a reasonable interpretation of multiple-prediction effects if one assumes either limited access to the memory stack or that the stack itself is of very limited capacity. If either the extent of a preliminary self-terminating scan or the range of introspective access is limited to two ordered elements, then the similarity of the first-second predic- 
tion type results to the most-least results would follow from the hypothesis that both second and least likely predictions indicated the contents of the same terminal stack position. A limited capacity or access interpretation is also consistent with the personal observation that we have been unable to train subjects to generate more than two predictions per trial within reasonable time constraints.

Finally, Lupker and Theios (1975) and Theios et al. (1973) obtained independent support for a limited capacity stack in which the number of elements had been treated as a model parameter. One limitation of their analyses (suggested by Lupker and Theios) is that alternative models of stimulus identification may be rejected, not because of flaws in the serial scanning assumptions or actual capacity limitations, but because of incorrect specification of the stack reordering process. Hacker and Hinrichs (1974) reported prediction protocols incompatible with proposed ordering mechanisms. An advantage of the verbal prediction paradigm is that stimulus prediction analyses are not dependent upon the specification of reordering axioms. As an alternative source of order information, verbal predictions may provide a useful converging operation for the investigation of proposed serial self-terminating processes.

\section{REFERENCE NOTE}

1. Theios, J., \& Falmagne, J. C. A probabilistic pushdown stack model for the organization of short-term memory in stimulus identification and prediction tasks. Paper presented at the Mathematical Psychology meetings, Princeton, New Jersey, September 1971.

\section{REFERENCES}

Acosta, E., Jn. Selective preparation: A comparison of cues and predictions in rapid decision-making. Unpublished doctoral dissertation, University of Iowa, 1977.

Bernstein, I. H., \& ReEse, C. Choice reaction time and behavioral hypotheses: The effects of learning sequential redundancies. Psychonomic Science, 1967, 9, 189-190.

Estes, W. K. Probability learning. In A. W. Melton (Ed.), Categories of human learning. New York: Academic Press, 1964.

Geller, E. S. Prediction outcome and choice reaction time: Inhibition versus facilitation effects. Acta Psychologica, 1975, 39, 69-82.

Geller, E. S., \& Pitz, G. F. Effects of prediction, probability, and run length on choice reaction speed. Journal of Experimental Psychology, 1970, 84, 361-367.

Hacker, M. J., \& Hinrichs, J. V. Multiple predictions in choice reaction time: A serial memory scanning interpretation. Journal of Experimental Psychology, 1974, 103, 999-1005.

Hinrichs, J. V. Probability and expectancy in two-choice reaction time. Psychonomic Science, 1970, 21, 227-228.

HinRICHS, J. V., \& CrafT, J. L. Verbal expectancy and probability in two-choice reaction time. Journal of Experimental Psychology, 1971, 88, 367-371.

HinRICHS, J. V., \& KRAINz, P. L. Expectancy in choice reaction time: Anticipation of stimulus or response? Journal of Experimental Psychology, 1970, 85, 330-334.

KEELE, S. W. Repetition effect: A memory-dependent process. Journal of Experimental Psychology, 1969, 80, 243-248.

LUPKER, S. J., \& ThEios, J. Tests of two classes of models for choice reaction times. Journal of Experimental Psychology: Human Perception and Performance, 1975, 104, 137-146.

Shiffrin, R. S., \& Schneider, W. An expectancy model for memory search. Memory \& Cognition, 1974, 2, 616-628.

STERnBERG, S. High-speed scanning in human memory. Science, $1966,153,652-654$.

Theios, J., Smith, P. G., Haviland, S. E., Traupmann, J., \& Moy, M. C. Memory scanning as a serial, self-terminating process. Journal of Experimental Psychology, 1973, 97, 323-336.

WICKElgREN, W. A. Speed-accuracy tradeoff and information processing dynamics. Acta Psychologica, 1977, 41, 67-85.

Williams, J. A. Sequential effects in disjunctive reaction time: Implications for decision models. Journal of Experimentol Psychology, 1966, 71, 665-672.

YELLOTT, J. I. Correction for fast guessing and the speed-accuracy tradeoff in choice reaction time. Journal of Mathematical Psychology, 1971, 8, 159-199.

\section{NOTE}

1. Within- and/or between-subjects mixtures of RT distributions resulting from uncontrolled shifts of attention to the unpredicted stimulus in the least likely, two-stimulus condition should be reflected in an increase in the within- and/or betweensubjects variability of the RTs in comparison with the most likely condition. There was a nonsignificant but positive trend between subjects, and the mean log variability within the individual RT distributions was marginally higher in the least than in the most likely conditions $[F(1,11)=9.40, p<.05]$. Although the scanning model leads to a number of nonparametric predictions concerning higher moments and distributional properties (Hacker \& Hintichs, 1974; Lupker \& Theios, 1975), there were insufficient data to seriously support such analyses in the present study.

(Received for publication December 7, 1978; revision accepted July $31,1979$. 